\title{
Decay mechanism and lifetime of ${ }^{67} \mathrm{Kr}$
}

\author{
L. V. Grigorenko, ${ }^{1,2,3}$ T. A. Golubkova, ${ }^{4}$ J. S. Vaagen, ${ }^{5}$ and M. V. Zhukov ${ }^{6}$ \\ ${ }^{1}$ Flerov Laboratory of Nuclear Reactions, JINR, RU-141980 Dubna, Russia \\ ${ }^{2}$ National Research Nuclear University “MEPhI”, Kashirskoye shosse 31, RU-115409 Moscow, Russia \\ ${ }^{3}$ National Research Centre "Kurchatov Institute”, Kurchatov sq. 1, RU-123182 Moscow, Russia \\ ${ }^{4}$ Advanced Educational and Scientific Center, Moscow State University, Kremenchugskaya 11, RU-121357 Moscow, Russia \\ ${ }^{5}$ Institute of Physics and Technology, University of Bergen, N-5007 Bergen, Norway \\ ${ }^{6}$ Department of Physics, Chalmers University of Technology, S-41296 Göteborg, Sweden
}

(Received 18 December 2016; published 21 February 2017)

\begin{abstract}
The lifetime of the recently discovered $2 p$ emitter ${ }^{67} \mathrm{Kr}$ was found to be considerably below the lower limit predicted theoretically. This communication addresses this issue. Different separation energy systematics are analyzed and different mechanisms for $2 p$ emission are evaluated. We find that the most plausible reason for this disagreement is the decay mechanism of ${ }^{67} \mathrm{Kr}$, which is not "true $2 p$ " emission but rather "transitional dynamics" on the borderline between true $2 p$ and sequential $2 p$ decay mechanisms. If this is correct, this imposes stringent limits of $E_{r}=1.35-1.42 \mathrm{MeV}$ on the ground-state energy of ${ }^{66} \mathrm{Br}$ relative to the ${ }^{65} \mathrm{Se}-p$ threshold.
\end{abstract}

DOI: 10.1103/PhysRevC.95.021601

Introduction. Discovery of a new case of two-proton $(2 p)$ radioactive decay has been reported recently in ${ }^{67} \mathrm{Kr}$ [1]. This is an important advance in the field because ${ }^{67} \mathrm{Kr}$ is the heaviest $2 p$ emitter observed so far, thus providing new opportunities for refining our understanding of the $2 p$ radioactivity phenomenon. For relatively small $2 p$ decay energy (found to be $E_{T}=1690 \pm 17 \mathrm{keV}$ ) the $2 p$ decay branch is in tough competition with weak transitions, providing only $37 \pm 14 \%$ of the decay probability. The measured total and partial $2 p$ lifetimes are $7.4 \pm 3.0 \mathrm{~ms}$ and $20 \pm 11 \mathrm{~ms}$ respectively. The previous (2003, [2]) theoretical predictions within the three-body cluster model provided a lower limit of the lifetime $240_{-70}^{+100} \mathrm{~ms}$ (within experimental decay energy uncertainty). Here, we are going to make refined theoretical calculations and discuss possible origins of the observed discrepancy.

Two-proton radioactivity predicted in 1960 [3] was experimentally discovered in ${ }^{45} \mathrm{Fe}$ in $2002[4,5]$ after four decades of dedicated search. Since that time our knowledge in this field has expanded tremendously. Radioactive $2 p$ decay has been found in ${ }^{19} \mathrm{Mg},{ }^{48} \mathrm{Ni}$, and ${ }^{54} \mathrm{Zn}$, and later the first information about two-proton correlations was obtained for these isotopes [6]. Highly precise information about three-body correlations is available for ${ }^{6} \mathrm{Be}[7-9]$ and ${ }^{16} \mathrm{Ne}[10,11]$. Evidence has been obtained that the $2 p$ decay of ${ }^{30} \mathrm{Ar}$ is of the "transitional type" with a decay mechanism on the borderline between true $2 p$ decay and sequential $2 p$ decay $[12,13]$.

The theoretical description of the $2 p$ emission based on the core $+p+p$ three-cluster model [14] has achieved a high level of sophistication [6]. The lifetimes for a number of $2 p$ emitters were successfully predicted $[2,6]$. The predicted connections between configuration mixing in the wave function (WF) structure and correlations in $2 p$ decay were experimentally confirmed $[15,16]$. Detailed comparison of the theoretical and observed correlations for the lightest $2 p$ emitters ${ }^{6} \mathrm{Be}$ and ${ }^{16} \mathrm{Ne}$ demonstrated high accuracy of the approach $[7,8,10,11]$. The studies of [10] demonstrated the ability to describe very delicate long-range effects of the three-body Coulomb continuum problem. The predictions of the three-body approach have so far been reliable and therefore it is important to understand what went wrong in the ${ }^{67} \mathrm{Kr}$ case.

There are several possible reasons why the lifetime could have been overestimated in our previous three-body calculations [2]:

(i) Slow convergence was demonstrated for three-body calculations when the two-body resonance energy $E_{r}$ becomes sufficiently low and enters the two-proton decay energy window from above: $E_{r}>0.84 E_{T}, E_{r} \rightarrow 0.84 E_{T}$. This problem was overcome in the later (2007) studies $[17,18]$ focusing on

${ }^{17} \mathrm{Ne}$ and ${ }^{45} \mathrm{Fe}$ cases. However, the predictions for heavier systems were not revised.

(ii) The "standard" systematics of potential radii $a=$ $r_{0}\left(A_{\text {core }}+1\right)^{1 / 3}$, where $r_{0}=1.2 \mathrm{fm}$, was used in the pioneering studies [2]. For a nuclear system as heavy as ${ }^{67} \mathrm{Kr}$, the sensitivity of lifetime to this choice could be more important than for the lighter systems. Some additional adjustment of the single-particle orbital properties of the calculations is highly desirable.

(iii) Logical continuation of the assumption in item (i) is to decrease further the $E_{r}$ value to the range $0.8 E_{T}<E_{r}<$ $0.84 E_{T}$. The phenomenon which can drastically increase the width and thus decrease the lifetime of a $2 p$ emitter is a transition from true $2 p$ to sequential $2 p$ decay mechanisms. Our studies have shown that this question is especially important for ${ }^{67} \mathrm{Kr}$ since various estimate results point to an energy region for $E_{r}$ which does not allow us to exclude such a possibility.

In this work we repeated calculations [2] for ${ }^{67} \mathrm{Kr}$ with technical improvements. We performed separation energy evaluations, introduced better restricted core- $p$ interactions, and performed the ${ }^{67} \mathrm{Kr}$ lifetime studies both in $R$-matrix semianalytical and full three-body decay models. We conclude that variant (iii) seems to be the most plausible explanation of the situation.

Width estimates within improved direct decay model. The semianalytical $R$-matrix direct decay model of $2 p$ decay is a convenient tool for the simple evaluation of $2 p$ lifetimes 


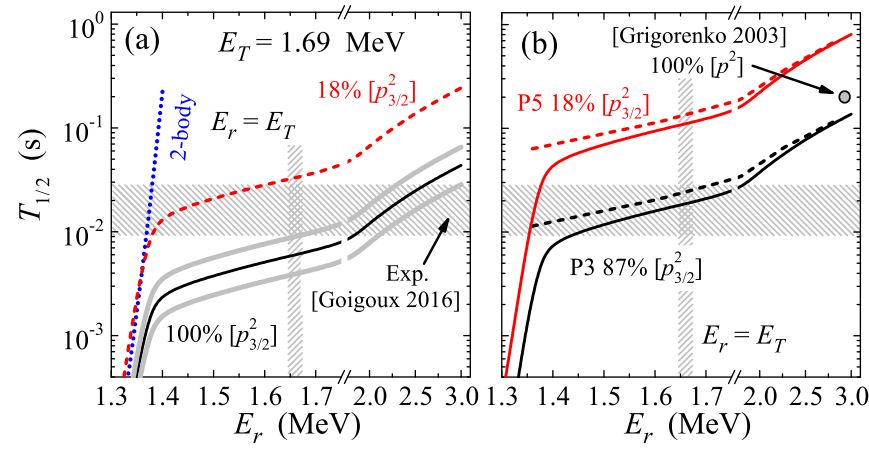

FIG. 1. Lifetime of ${ }^{67} \mathrm{Kr}$ as a function of the $p$-wave ground-state resonance energy $E_{r}$ in ${ }^{66} \mathrm{Br}$ for fixed energy $E_{T}=1.69 \mathrm{MeV}$. (a) IDDM model. Solid black and dashed red curves correspond to different assumed weights of the $\left[p^{2}\right]$ configuration. Solid gray curves correspond to the solid black curve with $\pm 0.2 \mathrm{fm}$ modified channel radius $r_{\text {cp }}$. The blue dotted line shows the two-body $R$-matrix estimate of the decay width into the ${ }^{66} \mathrm{Br}+p$ channel with energy $E_{T}-E_{r}$. (b) Dashed lines show three-body model results for P3 and P5 potentials producing different configuration mixing. Solid lines show the extrapolation of three-body results from large $E_{r}$ values (where they are converged) by IDDM curves. The referred publications are [1] for [Goigoux 2016] and [2] for [Grigorenko 2003].

and systematic studies $[6,17,18]$. This model works well when decay via a single quantum configuration is dominating and the nucleon-nucleon final-state interaction can be neglected. For ground-state decays, the three-body width $\Gamma$ within this model depends just on three parameters $\left(E_{T}, E_{r}, \Gamma_{r}\right)$ and an angular momentum coupling scheme.

To evaluate the effect of the low-lying states of ${ }^{66} \mathrm{Br}$ on the width of ${ }^{67} \mathrm{Kr}$ in this work we use the improved direct decay model (IDDM) of [13], which utilizes a more complex semianalytical approximation and is better tuned phenomenologically. The IDDM lifetimes of ${ }^{67} \mathrm{Kr}$ are shown in Fig. 1(a) as functions of the ${ }^{66} \mathrm{Ga}$ ground state (g.s.) energy. The g.s. of ${ }^{66} \mathrm{Ga}$ has $J^{\pi}=0^{+}$. This means that it is likely to involve a $p_{3 / 2}$ single-particle state coupled to the ${ }^{65} \mathrm{Se}$ g.s. with $J^{\pi}=3 / 2^{-}$. So, we assume $\left[p_{3 / 2}^{2}\right]_{0}$ for the ${ }^{67} \mathrm{Kr}$ g.s. decay. The width calculations for such a heavy $2 p$ emitter are very sensitive to the properties of the Coulomb barrier. The $R$-matrix channel radius $r_{\mathrm{cp}}=6.12 \mathrm{fm}$ and the reduced width $\theta^{2}=1$ are chosen to reproduce exactly the width of the resonance obtained with potential P1 (discussed below). Small variation of the value $r_{\mathrm{cp}}$ by $\pm 0.2 \mathrm{fm}$ leads to more than a factor of 2 variation of the lifetime, see gray curves in Fig. 1(a). The shell-model calculations of Ref. [1] predicted that the weight of the $\left[p_{3 / 2}^{2}\right]$ configuration in ${ }^{67} \mathrm{Kr}$ is only $\sim 18 \%$, which implies a corresponding increase of the lifetime predicted in the IDDM (dashed curve).

Two conclusions can be made from these calculations: (a) The transition "true $2 p$ " $\rightarrow$ "sequential $2 p$ " is taking place in ${ }^{67} \mathrm{Kr}$ in the $E_{r}=1.35-1.42 \mathrm{MeV}$ range $\left(S_{p}\right.$ from -340 to $-270 \mathrm{keV}$ ). (b) For the pure $\left[p_{3 / 2}^{2}\right]$ structure of

${ }^{67} \mathrm{Kr}$ the calculated lifetime is consistent with experiment for quite a broad range of $E_{r} \sim 1.7-2.7 \mathrm{MeV}$. In contrast, for

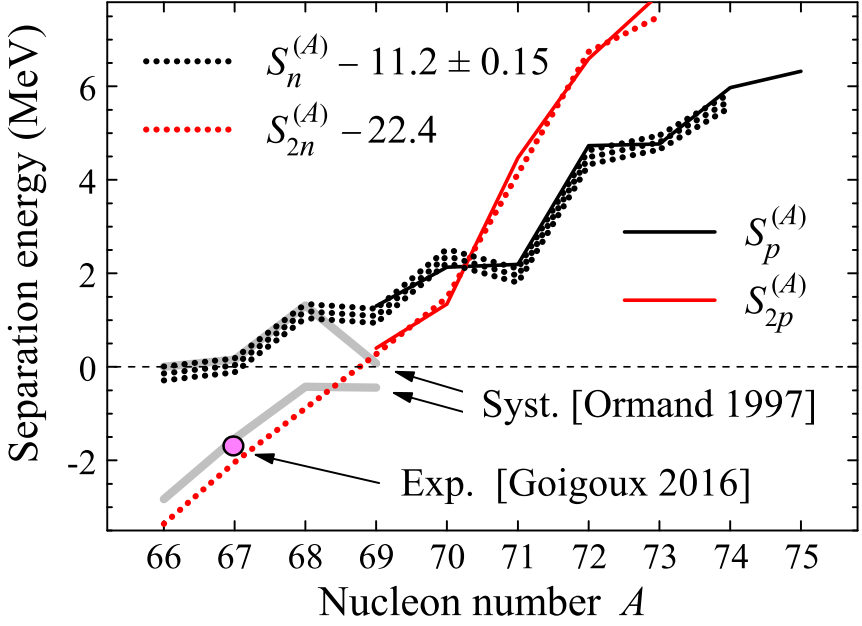

FIG. 2. Systematics of $N$ and $2 N$ separation energies $\left(S_{N}^{(A)}\right.$ and $S_{2 N}^{(A)}$, respectively) for krypton isobar and mirror isotone members with mass number $A$. The isotone values are provided with constant offsets (in $\mathrm{MeV}$ ) for visual comparison. Thick gray lines marked as [Ormand 1997] show systematics predictions from Ref. [19].

realistic shell-model structure $\left(\sim 18 \%\right.$ of $\left.\left[p_{3 / 2}^{2}\right]\right)$, the calculated lifetimes agree with experiment only in a much narrower range $E_{r} \sim 1.38-1.58 \mathrm{MeV}$ strongly overlapping with a "transitional dynamics" range. Below we try to understand how realistic is the latter possibility.

Systematic consideration of separation energies. It is necessary to clarify the decay mechanism of ${ }^{67} \mathrm{Kr}$ in order to clarify the problem of the ${ }^{67} \mathrm{Kr}$ lifetime. For the decay mechanism the question about the relation of $p$ and $2 p$ separation energies $S_{p}$ and $S_{2 p}$ is decisive [13]. We try three different types of estimates for these values.

Figure 2 shows the systematics of $N$ and $2 N$ separation energies (SSE) for krypton isobar and mirror isotone. The energy trends of the isotone and isobar nicely overlap in the mass range, where both of them are experimentally known. Extrapolation provided for $S_{2 p}$ agrees very well with data [1] and systematics studies [19]. The extrapolation for $S_{p}$ seems to be more uncertain and points to $S_{p}=30 \pm 150 \mathrm{keV}$ (also in a good agreement with [19]). However, an extra binding of just $200-300 \mathrm{keV}$ is needed to get into the transitional regime and it is known that the Thomas-Ehrman shift (TES) can easily modify this value to more negative values $[12,20]$.

Figure 3 shows the systematics of experimental odd-even staggering (OES) energies $2 E_{\mathrm{OES}}=S_{2 N}^{(A)}-2 S_{N}^{(A-1)}$ for the krypton isobar and mirror isotone. If we use the extrapolated value $2 E_{\mathrm{OES}}=2 \mathrm{MeV}$ then $S_{p}=200 \mathrm{keV}$ is obtained. However, the TES effect strongly changes this systematics [12,20]. If we take reasonable $2 E_{\mathrm{OES}}=1 \mathrm{MeV}$ then $S_{p}=-340 \mathrm{keV}$ is obtained, which corresponds to decay dynamics deeply in the transitional region.

The third estimate is a direct $S_{p}$ evaluation in a potential model which explicitly contains the TES. For this estimate two parameters are essential: potential radius and charge radius of the core. We take a Woods-Saxon potential with "standard" systematic parameters (P1, see Table II) $a=$ $r_{0}\left(A_{\text {core }}+1\right)^{1 / 3}=4.85 \mathrm{fm}$ and diffuseness $d=0.65 \mathrm{fm}$. The 


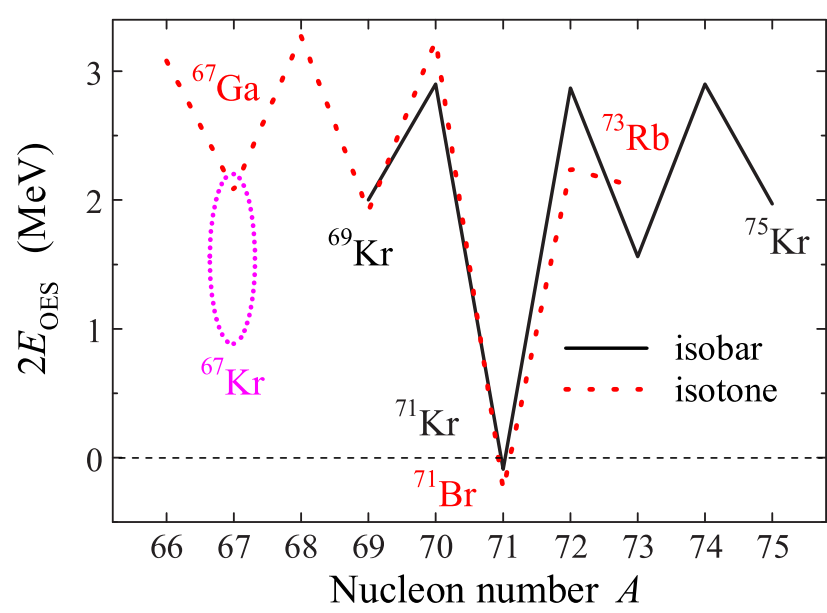

FIG. 3. Systematics of odd-even staggering energies $E_{\mathrm{OES}}$ for krypton isobar and mirror isotone. Dotted ellipsis show the expected uncertainty for ${ }^{67} \mathrm{Kr}$ due to the TES.

experimental systematics of charge radii [21] for krypton and selenium isotopes are shown in Fig. 4. We can expect both falling and rising trends when approaching the proton drip line. Thus we take a relatively broad range $r_{\mathrm{ch}}=4.0$ $4.21 \mathrm{fm}$ for ${ }^{65} \mathrm{Se}$. The corresponding Coulomb potential of a homogeneously charged sphere is used with radius $r_{\text {sph }}$ defined as $r_{\mathrm{sph}}^{2}=(5 / 3)\left[r_{\mathrm{ch}}^{2}+0.8^{2}\right]$. The $S_{p}$ calculated in a single-particle potential model based on this uncertainty of the charge radius ranges from $S_{p}=-320$ to $S_{p}=-80 \mathrm{keV}$.

The results of all estimates are summarized in Table I. We see here that various approaches provide $S_{p}$ values with uncertainties, which does not exclude the possibility of the transitional type of decay mechanism.

Three-body decay calculations. The core $+p+p$ cluster model of $2 p$ radioactivity is based on the solution of a three-

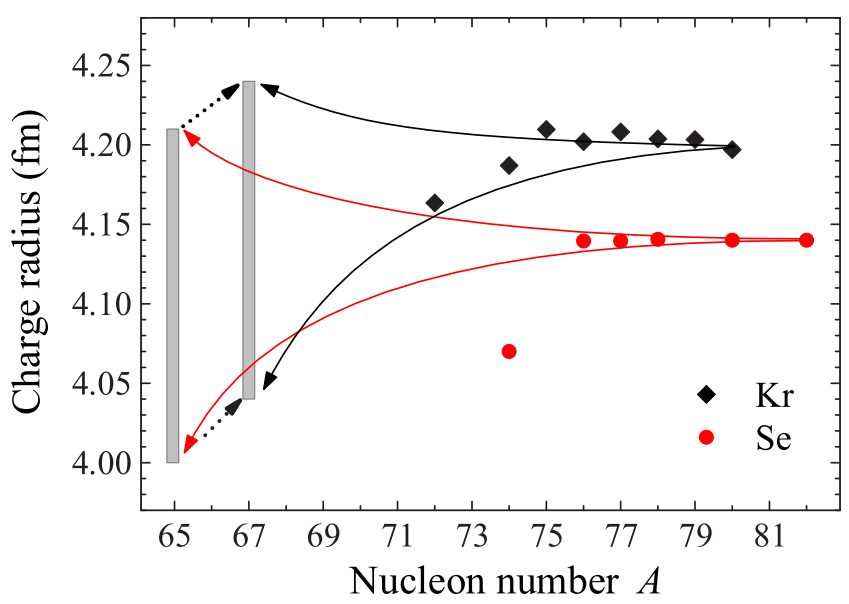

FIG. 4. Systematics of charge radii for krypton and selenium isobars. Solid arrows extrapolate rising and falling trends along the isotopic chains which could be expected based on data on other nuclei. Gray rectangle shows expected uncertainty for ${ }^{65} \mathrm{Se}$. The dashed arrows translate it into ${ }^{67} \mathrm{Kr}$ charge radius uncertainty by using an independent particle model, which seems consistent with analogous extrapolation for the krypton isobaric chain.
TABLE I. Comparison of range of $S_{p}$ values (in $\mathrm{keV}$ ) in ${ }^{67} \mathrm{Kr}$. The first column shows the $S_{p}$ range for transition "true $2 p$ " $\rightarrow$ "sequential $2 p$ " decay mechanisms. The other columns show results of different systematic evaluations of the upper and lower boundaries for the $S_{p}$ value.

\begin{tabular}{lccrrc}
\hline \hline & Transition & Ref. [19] & SSE & OES & TES \\
\hline Upper & -290 & 130 & 180 & 200 & -80 \\
Lower & -340 & -440 & -120 & -340 & -320 \\
\hline \hline
\end{tabular}

body Schrödinger equation with complex energy and pure outgoing wave boundary conditions, within the hyperspherical harmonics $(\mathrm{HH})$ method:

$$
\begin{array}{r}
\left(\hat{H}_{3}-E_{T}+i \Gamma / 2\right) \Psi_{E_{T}}^{(+)}=0, \\
\hat{H}_{3}=\hat{T}_{3}+V_{p_{1-p_{2}}}+V_{\text {core- } p_{1}}+V_{\text {core- } p_{2}}+V_{3}(\rho),
\end{array}
$$

where $\hat{T}_{3}$ is the three-body kinetic energy, $V_{i j}$ are pairwise interactions between clusters, chosen based on available experimental information, and $V_{3}(\rho)$ is a phenomenological short-range potential depending only on a collective variable (hyperradius $\rho$ ) which is used to tune the total decay energy for the lifetime calculations. Equation (1) is solved using a kind of perturbative procedure. Real-energy equations are solved as

$$
\left(\hat{H}_{3}-E_{T}\right) \Psi_{E_{T}}^{(+)}=i \Gamma / 2 \Psi_{E_{T}}^{(\text {box })}
$$

where $\Psi_{E_{T}}^{(\text {box })}$ is a real-energy eigenstate of the three-body Hamiltonian $\left(\hat{H}_{3}-E_{T}\right) \Psi_{E_{T}}^{\text {(box) }}=0$ with zero boundary condition at some large subbarrier radius. Solution is obtained for arbitrary three-body decay width $\Gamma$ value and then the actual $\Gamma$ value is defined using a so-called natural definition of the width $\Gamma=j / N$ ( $j$ is the outgoing flux via the hypersphere of large radius associated with $\mathrm{WF} \Psi_{E_{T}}^{(+)}$, while $N$ is the normalization of the WF inside this sphere). Such a procedure is very precise for the extremely small $\Gamma / E_{T}$ ratios typical of radioactive decays $[2,6]$.

In the $p$ - $p$ channel we use a semirealistic nucleon-nucleon potential [22]. The employed version of the $\mathrm{HH}$ method works with potentials without forbidden states. For that reason, when we turn to core- $p$ potentials, we need some substitute for potential P1 (Table II) used in the TES estimates above. In Ref. [2] the potential P2 was used together with the Coulomb potential of a charged sphere with radius $r_{\mathrm{sph}}=6.25 \mathrm{fm}$, which means an unrealistic charge radius and wrong TES systematics. For this work we produced the potential sets P3-P5, see Table III. Potential P3 was constructed to reproduce the systematics of the TES for potential P1 which means that

TABLE II. Parameters of Woods-Saxon potentials with surface (ls) interaction in the ${ }^{65} \mathrm{Se}-p$ channel. Energies are in $\mathrm{MeV}$, distances are in $\mathrm{fm}$.

\begin{tabular}{lccccccc}
\hline \hline Pot. & 1 & \multicolumn{1}{c}{$V_{c}$} & $a$ & $d$ & $V_{l s}$ & $r_{\text {sph }}$ & $r_{\mathrm{ch}}$ \\
\hline P1 & 1 & -50.485 & 4.85 & 0.65 & 1.5 & 5.5 & 4.21 \\
P2 & 1 & -20.89 & 4.825 & 0.65 & 0.5 & 6.248 & 4.82 \\
\hline \hline
\end{tabular}


TABLE III. Woods-Saxon potentials with volume $(l s)$ interaction and repulsive core in the ${ }^{65} \mathrm{Se}-p$ channel. Potential P5 has the same $l=1$ and Coulomb components as $\mathrm{P} 3$, so we give only the $l=3$ part of this potential.

\begin{tabular}{ccccccccc}
\hline \hline Pot. & 1 & $V_{1}$ & $a_{1}$ & $d_{1}$ & $V_{2}$ & $a_{2}$ & $d_{2}$ \\
\hline P3 & c & 1 & -26.389 & 5.0 & 0.65 & 75 & 1.5 & 0.53 \\
& ls & 1 & -1.0 & 5.0 & 0.65 & \multicolumn{2}{c}{$r_{\mathrm{sph}}=5.5 \mathrm{fm}$} \\
$\mathrm{P} 4$ & $\mathrm{c}$ & 1 & -57.612 & 4.55 & 0.65 & 150 & 2.7 & 0.53 \\
& s & 1 & -0.2 & 4.55 & 0.65 & $r_{\mathrm{sph}}=4.252 \mathrm{fm}$ \\
$\mathrm{P} 5$ & $\mathrm{c}$ & 3 & -45.8 & 5.0 & 0.65 & & \\
& ls & 3 & 0.2 & 4.55 & 0.65 & & & \\
\hline \hline
\end{tabular}

this WF has an analogous average orbital size. Potential P4 has the same behavior as P1 in the surface region and therefore practically the same resonance decay width. The ${ }^{66} \mathrm{Br}$ g.s. widths obtained with potentials P1-P5 are 1.18, 0.44, 0.74, 1.13 , and $0.74 \mathrm{eV}$, respectively.

The convergence of the three-body lifetime calculations is shown in Fig. 5. We use fully dynamic three-body calculations up to $K_{\max }=22$, while for the larger $K_{\max }$ values, the basis size is reduced to $K=22$ using the adiabatic procedure, see Ref. [16]. It can be seen that converged lifetime values are obtained for $E_{r}>2.2 \mathrm{MeV}$ at $K=60$. A value of $K \sim 100$ is required for convergence for $E_{r}>1.7 \mathrm{MeV}$. For lower $E_{r}$ values calculations are not converged: the decay dynamics is changing to sequential decay and the $\mathrm{HH}$ method is not suited for such situations. So, for this range of $E_{r}$ we use extrapolations by IDDM curves.

Lifetime calculations. The calculations with potential P3 of this work give considerably larger three-body decay widths than those with P2, used in [2]; see Fig. 5 and Table IV. This result can be explained by the larger orbital size for this potential, and, consequently, by larger two-body decay width, as pointed out above. With potential P4 we obtain $2 p$ width values which are about a factor of 2 larger than with P3. This difference is consistent with the simple estimate via squared ratio of two-body widths in the ${ }^{65} \mathrm{Se}-p$ channel $\left[\Gamma_{r}(\mathrm{P} 4) / \Gamma_{r}(\mathrm{P} 3)\right]^{2}=2.33$. We consider $\mathrm{P} 4$ set as too

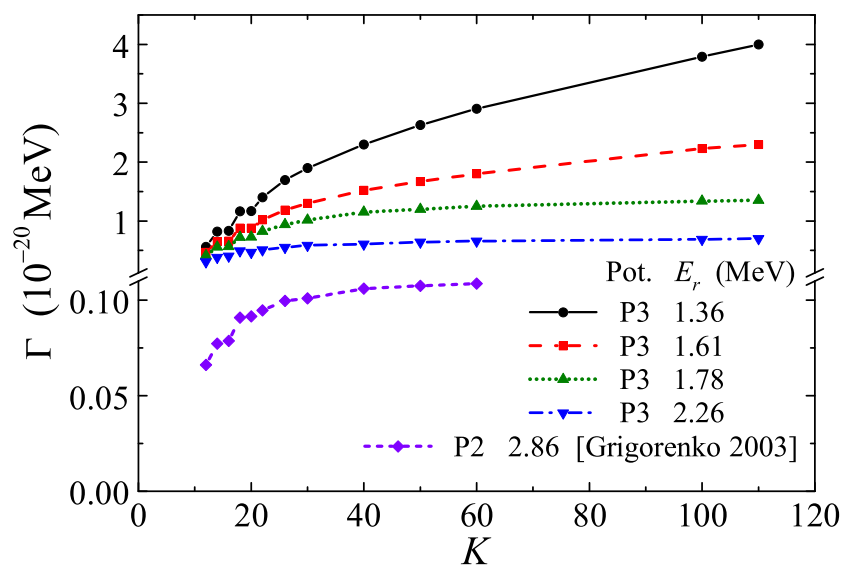

FIG. 5. Convergence of the three-body lifetime calculations as a function of the hyperspherical basis size $K_{\max }$. Potential P2 [Grigorenko 2003] is the one used in [2].
TABLE IV. Structure of the three-body WF $\Psi_{E_{T}}^{(+)}$in the internal region for valence protons. Weights of the shell-model-like configurations $\left[l_{j}^{2}\right]_{0}$ are given in percent. The last row shows shell-model (SM) predictions [1]

\begin{tabular}{lrccc}
\hline \hline Pot. & {$\left[p_{1 / 2}^{2}\right]_{0}$} & {$\left[p_{3 / 2}^{2}\right]_{0}$} & {$\left[f_{5 / 2}^{2}\right]_{0}$} & {$\left[f_{7 / 2}^{2}\right]_{0}$} \\
\hline P3 & 12.1 & 87.2 & 0.1 & 0.07 \\
P5 & 1.3 & 18.3 & 64.1 & 16.2 \\
SM & 13.8 & 17.6 & 67.2 & 2.4 \\
\hline \hline
\end{tabular}

unrealistic because of too large TES, and rely on P3 below. We would like to emphasize that a factor of 2 increase in the widths predicted for $\mathrm{P} 3$ is possible with tolerable modification of the single-particle WF geometry, and can be regarded as a measure of the theoretical uncertainty of our calculations. This factor of 2 is not large enough to modify major conclusions of this work. Potential set P5 contains interactions in $l=3$ and thus gives structure of ${ }^{67} \mathrm{Kr}$ with strong $p / f$ configuration mixing which is roughly consistent with shell-model structure predictions from [1].

The results of the three-body lifetime calculations as a function of energy $E_{r}$ for fixed $E_{T}$ are shown in Fig. 1(b) by dashed curves. Because the lifetime calculations with $E_{r}<1.7 \mathrm{MeV}$ are not converged, extrapolations to small $E_{r}$ values using IDDM calculations are performed (solid curves). The conclusion drawn here is the same as for IDDM: For a $\sim 100 \%\left[p^{2}\right]$ structure of ${ }^{67} \mathrm{Kr}$ (P3 potential) the agreement with experimental lifetime can be obtained for a broad range of $E_{r}$ values, while for a realistic ${ }^{67} \mathrm{Kr}$ structure (potential P5, $\sim 18 \%\left[p_{3 / 2}^{2}\right]$ ) the agreement is possible only in a narrow range of transitional $E_{r}$ values.

The transition from true $2 p$ to sequential decay lifetime is illustrated in Fig. 6 for different $E_{r}$ values. These are

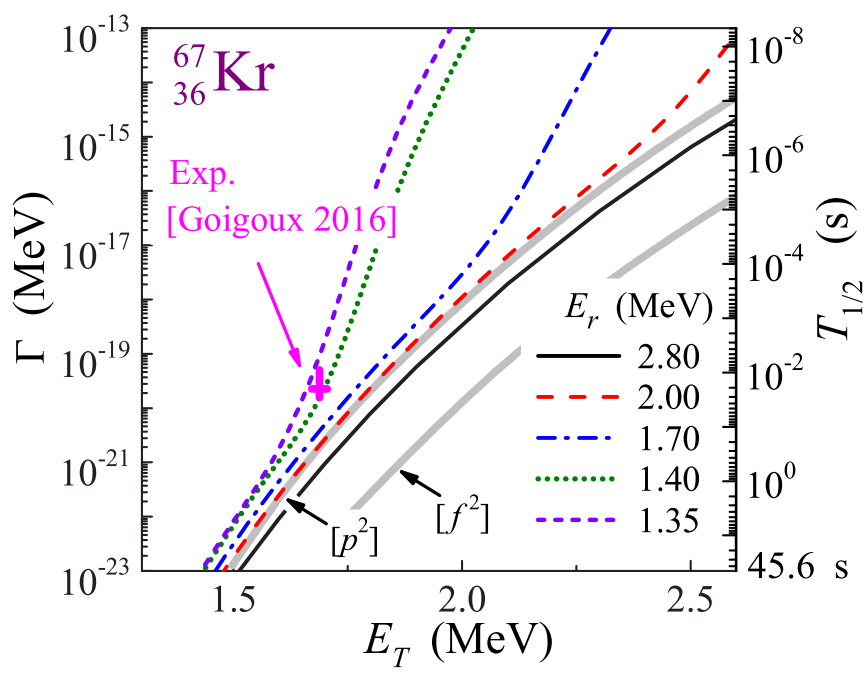

FIG. 6. Lifetime of ${ }^{67} \mathrm{Kr}$ as a function of $2 p$ decay energy $E_{T}$ for several $E_{r}$ values. The three-body model with realistic structure (P5 potential) and IDDM extrapolation were used. The results of the three-body calculations with pure $\left[l^{2}\right]$ configurations from [2] are shown by thick gray curves. The pink cross marked as [Goigoux 2016] shows the experimental value [1] with error bars. 
calculations with a realistic ${ }^{67} \mathrm{Kr}$ structure (P5 potential), performed at $E_{T}<0.7 E_{r}$ (where they are reliably converged) and extrapolated to higher $E_{T}$ values using the IDDM curves. Variation of $E_{r}$ is obtained by varying the charged sphere radius $r_{\mathrm{sph}}$ in the core- $p$ Coulomb potential.

Three-body correlations. As we have seen, the situation with separation energies in ${ }^{67} \mathrm{Kr}$ is very uncertain and there is a considerable chance that in this nuclide we face yet another example of transitional dynamics (we can probably exclude a possibility of the pure sequential decay mechanism, as this immediately leads to very short lifetimes). The answer to the question about the decay mechanism can be obtained by studies of energy correlations between the core and one of the protons. Figure 7 shows these correlations in the case of true $2 p$ decay and in the case of transitional decay dynamics "true $2 p$ " $\rightarrow$ "sequential $2 p$ " taking place in a very narrow interval of possible ${ }^{66} \mathrm{Br}$ g.s. energies. The probability of sequential decay changes from $\sim 5 \%$ at $E_{r}=1.35 \mathrm{MeV}$ to $\sim 95 \%$ at $E_{r}=1.40 \mathrm{MeV}$ together with more than an order-of-magnitude change in the lifetime (see Fig. 1). This effect would evidently be observable in modern experiments with time projection chambers $[15,23]$.

Conclusions. The discrepancy between the lifetimes predicted for ${ }^{67} \mathrm{Kr}$ in 2003 [2] and found in the recent measurements [1] inspired us to revisit the issue. We have reached the following conclusions:

(i) Various systematic studies favor for ${ }^{67} \mathrm{Kr}$ either a small positive or, which is more probable, a small negative $S_{p}$ value.

(ii) The experimentally observed lifetime of ${ }^{67} \mathrm{Kr}$ can be explained by the true $2 p$ decay mechanism, assuming dominance of the $\left[p_{3 / 2}^{2}\right]_{0}$ configuration in the structure of ${ }^{67} \mathrm{Kr}$. This will work only if the ${ }^{66} \mathrm{Br}$ g.s. is located close to or somewhat within the three-body decay "energy window," namely, if $E_{r}=1.45-2.0 \mathrm{MeV}$ ( $S_{p}$ from -240 to $310 \mathrm{keV}$ ).

(iii) If we take into account the realistic structure of ${ }^{67} \mathrm{Kr}$, predicted in $[1]\left(\sim 18 \%\right.$ of the $\left[p_{3 / 2}^{2}\right]_{0}$ configuration), then the only possible way to explain the lifetime is to consider a different decay mechanism. For $E_{r}=1.35-1.42 \mathrm{MeV}\left(S_{p}\right.$

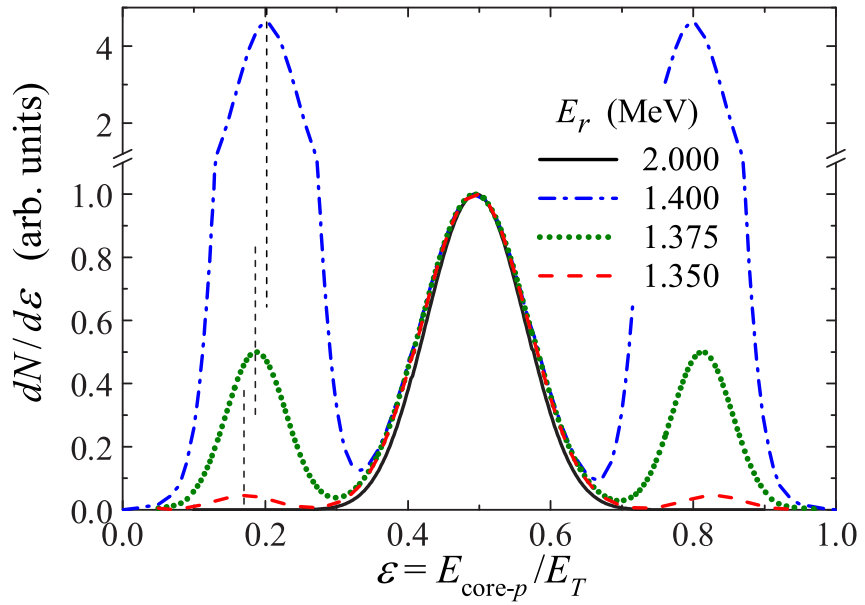

FIG. 7. Energy correlations between the ${ }^{65} \mathrm{Se}$ core and one of the protons in $2 p$ decay of ${ }^{67} \mathrm{Kr}$ with $E_{T}=1.69 \mathrm{MeV}$. Calculations by IDDM with different $E_{r}$ values in the core- $p$ subsystem ${ }^{66} \mathrm{Br}$ illustrate true $2 p$ decay mechanism $\left(E_{r}=2 \mathrm{MeV}\right)$ and the region of transitional decay dynamics $\left(E_{r}=1375-1400 \mathrm{keV}\right)$. The curves are normalized to unity maximum value of the $\varepsilon \sim 0.5$ peak. The estimated width of the ${ }^{66} \mathrm{Br}$ g.s. is very small, therefore we convolve sequential decay peaks with Gaussians of $150 \mathrm{keV}$ FWHM for the sake of visual comparison. Centroids of the low- $\varepsilon$ sequential decay peaks are indicated by the vertical dashed lines.

from -340 to $-270 \mathrm{keV}$ ) the decay of ${ }^{67} \mathrm{Kr}$ corresponds to a transitional dynamics on the borderline between true $2 p$ and sequential $2 p$ decay mechanisms. Further decrease of $E_{r}$ leads to a pure sequential decay mechanism with rapid decrease of the lifetime beyond the experimentally acceptable value, see Fig. 1(a).

(iv) The question about the decay mechanism of ${ }^{67} \mathrm{Kr}$ can be clarified by studies of $2 p$ correlations. Predicted effects are strong enough to be observable by modern correlation experiment, even if the counting rate is modest.
[1] T. Goigoux, P. Ascher, B. Blank, M. Gerbaux, J. Giovinazzo, S. Grévy, T. Kurtukian Nieto, C. Magron, P. Doornenbal, G. G. Kiss et al., Phys. Rev. Lett. 117, 162501 (2016).

[2] L. V. Grigorenko and M. V. Zhukov, Phys. Rev. C 68, 054005 (2003).

[3] V. I. Goldansky, Nucl. Phys. 19, 482 (1960).

[4] M. Pfützner, E. Badura, C. Bingham, B. Blank, M. Chartier, H. Geissel, J. Giovinazzo, L. V. Grigorenko, R. Grzywacz, M. Hellström et al., Eur. Phys. J. A 14, 279 (2002).

[5] J. Giovinazzo, B. Blank, M. Chartier, S. Czajkowski, A. Fleury et al., Phys. Rev. Lett. 89, 102501 (2002).

[6] M. Pfützner, M. Karny, L. V. Grigorenko, and K. Riisager, Rev. Mod. Phys. 84, 567 (2012).

[7] L. V. Grigorenko, T. D. Wiser, K. Mercurio, R. J. Charity, R. Shane, L. G. Sobotka, J. M. Elson, A. H. Wuosmaa, A. Banu, M. McCleskey et al., Phys. Rev. C 80, 034602 (2009).

[8] I. A. Egorova, R. J. Charity, L. V. Grigorenko, Z. Chajecki, D. Coupland, J. M. Elson, T. K. Ghosh, M. E. Howard,
H. Iwasaki, M. Kilburn et al., Phys. Rev. Lett. 109, 202502 (2012).

[9] A. Fomichev, V. Chudoba, I. Egorova, S. Ershov, M. Golovkov, A. Gorshkov, V. Gorshkov, L. Grigorenko, G. Kaminski, S. Krupko et al., Phys. Lett. B 708, 6 (2012).

[10] K. W. Brown, R. J. Charity, L. G. Sobotka, Z. Chajecki, L. V. Grigorenko, I. A. Egorova, Y. L. Parfenova, M. V. Zhukov, S. Bedoor, W. W. Buhro et al., Phys. Rev. Lett. 113, 232501 (2014).

[11] K. W. Brown, R. J. Charity, L. G. Sobotka, L. V. Grigorenko, T. A. Golubkova, S. Bedoor, W. W. Buhro, Z. Chajecki, J. M. Elson, W. G. Lynch et al., Phys. Rev. C 92, 034329 (2015).

[12] I. Mukha, L. Grigorenko, X. Xu, L. Acosta, E. Casarejos, W. Dominik, J. Duénas-Díaz, V. Dunin, J. Espino, A. Estradé et al., Phys. Rev. Lett. 115, 202501 (2015).

[13] T. Golubkova, X.-D. Xu, L. Grigorenko, I. Mukha, C. Scheidenberger, and M. Zhukov, Phys. Lett. B 762, 263 (2016). 
[14] L. V. Grigorenko, R. C. Johnson, I. G. Mukha, I. J. Thompson, and M. V. Zhukov, Phys. Rev. Lett. 85, 22 (2000).

[15] K. Miernik, W. Dominik, Z. Janas, M. Pfützner, L. Grigorenko, C. R. Bingham, H. Czyrkowski, M. Cwiok, I. G. Darby, R. Dabrowski et al., Phys. Rev. Lett. 99, 192501 (2007).

[16] L. V. Grigorenko, T. D. Wiser, K. Miernik, R. J. Charity, M. Pfützner, A. Banu, C. R. Bingham, M. Cwiok, I. G. Darby, W. Dominik et al., Phys. Lett. B 677, 30 (2009).

[17] L. V. Grigorenko and M. V. Zhukov, Phys. Rev. C 76, 014008 (2007).
[18] L. V. Grigorenko and M. V. Zhukov, Phys. Rev. C 76, 014009 (2007).

[19] W. E. Ormand, Phys. Rev. C 55, 2407 (1997).

[20] L. V. Grigorenko, T. A. Golubkova, and M. V. Zhukov, Phys. Rev. C 91, 024325 (2015).

[21] I. Angeli and K. Marinova, At. Data Nucl. Data Tables 99, 69 (2013).

[22] D. Gogny, P. Pires, and R. D. Tourreil, Phys. Lett. B 32, 591 (1970).

[23] P. Ascher, L. Audirac, N. Adimi, B. Blank, C. Borcea, B. A. Brown, I. Companis, F. Delalee, C. E. Demonchy, F. de Oliveira Santos et al., Phys. Rev. Lett. 107, 102502 (2011). 